\title{
Early-Warning for Environment-Economy Development Based on Boom Analysis in Dalian, China
}

\author{
Shasha Yang ${ }^{1, a}$, Ling $\mathrm{Xu}^{1, \mathrm{a}^{*}}, \mathrm{He}$ Wang $^{2, \mathrm{~b}}$ \\ ${ }^{1}$ School of Environmental Science and Technology, Dalian University of Technology, \\ Dalian 116024, China; \\ ${ }^{2}$ Liaoning Academy of Environmental Sciences, Shenyang 110031, China; \\ axuling@dlut.edu.cn, bwangh602@163.com
}

Keywords: environment-economy, early-warning system, boom analysis, Dalian.

\begin{abstract}
Environment-Economy development is a priority in China and it is supported by national policy. However, the study about measuring and warning regional development status of environment-economy is limited. In order to identify the impact of socio-economic development on the environment, we build an early-warning system to assess the current status of environment-economy development and predicate the future trend of this composite system. Our results show that the development status of environment-economy changed from boom decline to stable between 2005 and 2014, the overall status are in good condition, and the predicted results in next decade are in a stable state, but still need to guard against some indicators overheating.
\end{abstract}

\section{Introduction}

The early-warning research on the development of the environment-economy is developed from single field. Based on the synthetically consideration of the relationship between social economic activities and environment, the early-warning theory of environment-economy system is gradually formed. Some efforts have been taken to make early-warning analysis for the development of environment-economy previously. The most common is to set up the indicator system, and draw the comprehensive early-warning index for analysis [1, 2]. The common early-warning models such as multicriteria decision analysis [3], system dynamics [4] and so on. Wang [5] uses a single early-warning indicator of the elastic coefficient to make research of early-warning on regional environment-economy. In addition, as far as the current researches, most of them rely on specific categories, such as energy-environment-economy, forest-environment-economy and other complex systems [6, 7]. However, the above researches usually lacked of regional characteristics and neglected the interaction between environmental and economic system. Wang has built an early warning system based on booming analysis, which divided the indicators into three categories, to analyze the development of environment-economy by the diffusion index and composite index [8], which make up some shortcomings of the above methods. But this kind of research is only limited to the current time, and there is neither early-warning about the future development, nor the running state of development of environment-economy intuitively.

The objective of this paper is to develop an early-warning system of environmental and economic development based on the boom theory, and build a more comprehensive early-warning indicator system combined with regional characteristics. Moreover, the time series method based on BP neural network was used to predict the value of warning indicators in the next 10 years, and to address decision makers in the allocation of their resources, to maintain and improve the level of environment-economy development. The case study refers to Dalian, a region in northeast of China. 


\section{Materials and methods}

\subsection{Early-warning system}

The early-warning system is to incorporate the multiple environmental economic warning indicators as a comprehensive indicator through the method of data processing. Comparing the environmental economic warning line, we issued a different signal to the current environmental economic development status of indicators and the comprehensive indicator. Finally, by observing and analyzing the changes of the signal, to judge the current and future status of the environment-economy system. The early-warning system was divided into five intervals, which could reflect the status of environmental and economic development and with different signal lights respectively. Among them, the "red light" represents the boom of environmental and economic system is overheating and may be in a state of overload, and an internal tightening is required in this case; "yellow light" represents the boom of system is still steady, but heat transfer or ultra-stable is possible in short-term; "green light" represents the boom of environmental and economic system is stable and running well; "light blue light" represents the boom is decreased, but stable transfer or turn cold is possible in short-term; "blue light" represents the boom is shrinking and may be in a state of stagnation, which need to take strong measures to stimulate environmental and economic growth.

\subsection{Grey correlation analysis}

Grey theory is to study phenomenon with uncertainties whose inner information is not clear [9]. It quantitatively describes the relative change of the factors in the process of the system development, that is, the relativity of indicators such as the change of the size, direction, speed and others. In our case, the grey correlation analysis is used to determine the weight of indicators. The calculation steps of grey correlation analysis are as follows:

(1) Determination of the reference sequence. The reference sequence which represents system characteristics is expressed as $\left\{\mathrm{X}_{0}(\mathrm{t})\right\}=\left\{\mathrm{X}_{01}, \mathrm{X}_{02}, \ldots \ldots \mathrm{X}_{0 \mathrm{n}}\right\}$. In our case, the gross industrial output value $\left(\mathrm{X}_{0}\right)$ was used as the reference.

(2) Standardization of $\mathrm{X}_{0}$ and $\mathrm{X}_{\mathrm{i}}$. Data processing which makes dates dimensionless should be carried out before gray correlation analysis. That is,

$$
Y_{i}=\frac{X_{i}-x}{s}
$$

Among them, $Y_{i}$ is the value of each indicator after the standardized transformation, $X_{\tilde{i}}$ is the initial value of each indicator, $X X$ is the sample mean, and $\mathrm{S}$ is the sample standard deviation.

(3) Calculation of correlation coefficient. The correlation coefficient $\mathrm{L}_{0 \mathrm{i}}(\mathrm{t})$ between the reference sequence $\left\{\mathrm{X}_{0}(\mathrm{t})\right\}$ and the comparison sequence $\left\{\mathrm{X}_{\mathrm{i}}(\mathrm{t})\right\}$ can be calculated by the following formula:

$$
L_{0 i}=\frac{\Delta_{\min }+\Delta_{\max }}{\Delta_{\mathrm{oi}}(t)+\rho \Delta_{\max }}
$$

In the formula, $\Delta_{0 i}(t)$ represents the absolute difference of two comparison sequence at $t$ moments, $\Delta_{\max }$ and $\Delta_{\min }$ represents the maximum value and minimum value of the absolute difference of all comparison sequence each time respectively. $\rho$ is the resolution factor, used to increase the significant difference between correlation coefficient, usually take 0.1 0.5.

(4) Calculation of correlation degree. The correlation degree between the comparison sequence and reference sequence is calculated by the average value of its correlation coefficient each moment:

$$
r_{0 i}=\frac{1}{N} \sum_{t=1}^{N} L_{0 i}(t)
$$

In the formula, $r_{0 i}$ represents the correlation degree of comparison sequence $\mathrm{X}_{\mathrm{i}}$ and reference sequence $\mathrm{X}_{0}, N$ is the length of the comparison sequence (i.e. number of data).The software of data processing, DPS, was used to calculate the correlation degree of the data, and the correlation degree between each indicator and $\mathrm{X}_{0}$ is regarded as the weight of it.

\subsection{Time series method based on BP neural network}

In this paper, the time series method based on BP neural network is used to predict the values of early warning indicators. BP neural network is a kind of multi-layer feed forward networks [10]. It includes input layer, hidden layer and output layer. The main features of the network are the 
transmission signals forward and errors backward [11]. The software of Matlab 2014a was used to program and design BP neural network. For each indicator, the data of each adjacent 3 years was selected as a set of input, and the fourth year as the output. Three layers of BP neural network are used in our case, the input layer neuron number is 3 , the output layer neuron number is 1 , and the hidden layer neuron satisfies both the formula (4) and (5).

$$
m=\sqrt{w+n}+\mathrm{R}(10)
$$

In the formula, $m$ indicates the number of hidden neurons; $n$ and $w$ indicate the number of neurons in the input layer and the output layer; $\mathrm{R}(10)$ takes any number between $0 \sim 10$.

\section{Calculation}

\subsection{Indicators}

The early-warning indicator system of environment-economy development is comprehensive, systematic, which not only include the internal indicators of environment and economy, but also affected by the external factors such as social environment, environment-economy and other aspects. The social development, environment, economy, environment-economy and other aspects should be considered. According to the principle of indicator selection (Science, feasibility, hierarchy, completeness, dynamic) [12], expert opinion, and regional characteristics of Dalian city and other factors, 4 categories and 29 indicators were selected ultimately. (Table 1)

\begin{tabular}{|c|c|c|c|c|c|}
\hline Category & $\begin{array}{c}\text { Sub } \\
\text { category }\end{array}$ & Indicator & Category & $\begin{array}{c}\text { Sub } \\
\text { category }\end{array}$ & Indicator \\
\hline \multirow[t]{3}{*}{ Economy } & $\begin{array}{l}\text { Economic } \\
\text { developm- } \\
\text { ent }\end{array}$ & $\begin{array}{l}\text { Per capita GDP (X1) Per capita fiscal } \\
\text { revenue (X2) Consumer price index (X3) } \\
\text { Total social fixed investment (X4) }\end{array}$ & \multirow[t]{3}{*}{$\begin{array}{l}\text { Environ- } \\
\text { ment }\end{array}$} & $\begin{array}{l}\text { Pollution } \\
\text { emission }\end{array}$ & $\begin{array}{l}\text { Discharge of industrial waste } \\
\text { water (X11) Industrial } \mathrm{SO}_{2} \\
\text { emissions (X12) Industrial solid } \\
\text { waste production (X13) Industrial } \\
\text { smoke (dust) emissions (X14) } \\
\text { Severely polluted sea area near } \\
\text { the coast (X15) }\end{array}$ \\
\hline & $\begin{array}{l}\text { Economic } \\
\text { structure }\end{array}$ & $\begin{array}{l}\text { Second industry accounted for GDP (X5) } \\
\text { Third industry accounted for GDP (X6) }\end{array}$ & & \multirow[t]{2}{*}{$\begin{array}{l}\text { Environm } \\
\text {-ental } \\
\text { control }\end{array}$} & \multirow{2}{*}{$\begin{array}{l}\text { Discharge attainment rate of } \\
\text { industrial waste water (X16) } \\
\text { Removal rate of Industrial } \mathrm{SO}_{2} \\
\text { (X17)Disposal rate of Industrial } \\
\text { solid waste (X18) Removal rate } \\
\text { of Industrial smoke (dust) (X19) }\end{array}$} \\
\hline & $\begin{array}{l}\text { Economic } \\
\text { benefit }\end{array}$ & $\begin{array}{l}\text { Industrial added value (X7) Total social } \\
\text { labor productivity (X8) Average annual } \\
\text { balance of current assets (X9) Tax rate of } \\
\text { output value (X10) }\end{array}$ & & & \\
\hline $\begin{array}{l}\text { Environ- } \\
\text { ment } \\
\text { economy }\end{array}$ & $\begin{array}{l}\text { Environm } \\
\text {-ent } \\
\text { economy }\end{array}$ & $\begin{array}{l}\text { Waste water discharge of ten thousand } \\
\text { yuan output value (X20) } \mathrm{SO}_{2} \text { emission of } \\
\text { ten thousand yuan output value (X21) } \\
\text { Industrial solid waste production of ten } \\
\text { thousand yuan output value (X22) Smoke } \\
\text { (dust) emissions of ten thousand Yuan } \\
\text { output value (X23) Urban environmental } \\
\text { investment accounted for GDP (X24) }\end{array}$ & Society & $\begin{array}{l}\text { Social } \\
\text { security }\end{array}$ & $\begin{array}{l}\text { Natural population growth rate } \\
\text { (X25) Per capita housing area } \\
\text { (X26) Per capita living } \\
\text { electricity consumption（X27） } \\
\text { Unemployment rate（X28） } \\
\text { Hospital beds of one thousand } \\
\text { people（X29） }\end{array}$ \\
\hline
\end{tabular}

Table 1 Early-warning indicator system

\subsection{Determine the limits of early-warning indicators}

In this paper, mathematical statistics method is used to determine the boundaries of early-warning indicators, which will be divided into five sections based on the boom theory. According to the principle of statistics, the value of random variable follows the normal distribution. Referring to the previous research experience [13] and the characteristics of the environment-economy development in Dalian City, chose $\left(\mu \pm_{\sigma}\right)$ as the boom normal interval, $\left(\mu \pm_{\sigma}, \mu \pm_{2 \sigma}\right)$ as the boom steady and boom decreasing interval respectively, deviate $\pm_{2} \sigma$ from the expected $\mu$ outside were the boom overheating and the boom shrinking interval respectively. Limits of some indicators were adjusted 
refer to previous research, expert consultation and current situation of Dalian's environmental economic development. The limits of early-warning indicators see Table 2.

Table 2 The limits of early-warning indicators

\begin{tabular}{|l|c|c|c|c|c|c|c|c|c|}
\hline & Red $\leftarrow$ & Yellow $\leftarrow$ & Green $\leftarrow$ & Light Blue & & Red $\leftarrow$ & Yellow & Green $\leftarrow$ & Light Blue \\
\hline $\mathbf{X 1}$ & 125538 & 100486 & 50383 & 25331 & $\mathbf{X 1 6}$ & 98.52 & 97.60 & 95.76 & 94.84 \\
\hline $\mathbf{X 2}$ & 14734.0 & 11527.9 & 5115.7 & 1909.6 & $\mathbf{X 1 7}$ & 81.80 & 70.28 & 47.25 & 35.73 \\
\hline $\mathbf{X 3}$ & 106.6 & 104.8 & 101.3 & 99.5 & $\mathbf{X 1 8}$ & 99.87 & 97.85 & 95.31 & 93.29 \\
\hline $\mathbf{X 4}$ & 7705.0 & 5745.8 & 2806.7 & 847.3 & $\mathbf{X 1 9}$ & 99.35 & 98.24 & 96.58 & 95.47 \\
\hline $\mathbf{X 5}$ & 52.5 & 50.9 & 47.5 & 45.8 & $\mathbf{X 2 0}$ & 17.82 & 12.96 & 5.68 & 0.83 \\
\hline $\mathbf{X 6}$ & 46.6 & 44.9 & 41.7 & 40.0 & $\mathbf{X 2 1}$ & 0.0041 & 0.0032 & 0.0015 & 0.0006 \\
\hline $\mathbf{X 7}$ & 4078.2 & 3123.6 & 1214.4 & 259.8 & $\mathbf{X 2 2}$ & 0.1267 & 0.1098 & 0.0760 & 0.0591 \\
\hline $\mathbf{X 8}$ & 30.68 & 24.91 & 13.36 & 7.58 & $\mathbf{X 2 3}$ & 0.0021 & 0.0016 & 0.0006 & 0.0001 \\
\hline $\mathbf{X 9}$ & 6185.7 & 4840.7 & 2150.8 & 805.8 & $\mathbf{X 2 4}$ & 3.06 & 2.64 & 1.81 & 1.39 \\
\hline $\mathbf{X 1 0}$ & 21.68 & 16.34 & 8.33 & 4.32 & $\mathbf{X 2 5}$ & 4.20 & 2.43 & -1.10 & -2.86 \\
\hline $\mathbf{X 1 1}$ & 42921.51 & 37568.55 & 26862.61 & 21509.64 & $\mathbf{X 2 6}$ & 40.34 & 35.49 & 25.79 & 20.94 \\
\hline $\mathbf{X 1 2}$ & 13.64 & 11.92 & 8.47 & 6.75 & $\mathbf{X 2 7}$ & 703.7 & 639.6 & 511.6 & 447.5 \\
\hline $\mathbf{X 1 3}$ & 700.87 & 567.24 & 299.99 & 166.37 & $\mathbf{X 2 8}$ & 3.28 & 3.01 & 2.47 & 2.21 \\
\hline $\mathbf{X 1 4}$ & 8.44 & 6.69 & 3.21 & 1.46 & $\mathbf{X 2 9}$ & 6.92 & 6.09 & 4.42 & 3.58 \\
\hline $\mathbf{X 1 5}$ & 1804 & 1138 & 339 & 52 & & & & & \\
\hline
\end{tabular}

\subsection{Determine the limits of comprehensive early-warning index}

Due to the different impact of the indicators on the system, so the comprehensive early-warning index should calculate according to the weight of each indicator (Table 3). According to the limits division of indicators, score for five lights respectively, "red light" means five scores, "yellow light" means four scores, "green light" means three scores, "light blue light" means two scores, "blue light" means one score. The score of indicator is determined by the interval of the actual value belong to.

$$
E=\sum_{i=1}^{n} E_{i} \times X_{i}
$$

In the formula, $E$ represents the comprehensive early-warning index, $n$ represents the number of indicators, $E_{i}$ represents the point of indicator $i, X_{i}$ represents the weight of indicator $i$.

Table 3 The weight of indicators

\begin{tabular}{|c|c|c|c|c|c|c|c|c|c|c|}
\hline Indicator & $\mathrm{X} 1$ & $\mathrm{X} 2$ & $\mathrm{X} 3$ & $\mathrm{X} 4$ & $\mathrm{X} 5$ & $\mathrm{X} 6$ & $\mathrm{X} 7$ & $\mathrm{X} 8$ & $\mathrm{X} 9$ & $\mathrm{X} 10$ \\
\hline Weight & 0.9687 & 0.9205 & 0.7099 & 0.9307 & 0.8019 & 0.6429 & 0.9243 & 0.8751 & 0.9285 & 0.7658 \\
\hline Indicator & $\mathrm{X} 11$ & $\mathrm{X} 12$ & $\mathrm{X} 13$ & $\mathrm{X} 14$ & $\mathrm{X} 15$ & $\mathrm{X} 16$ & $\mathrm{X} 17$ & $\mathrm{X} 18$ & $\mathrm{X} 19$ & $\mathrm{X} 20$ \\
\hline Weight & 0.6255 & 0.7188 & 0.8994 & 0.8000 & 0.7230 & 0.7089 & 0.6218 & 0.8499 & 0.6424 & 0.6280 \\
\hline Indicator & $\mathrm{X} 21$ & $\mathrm{X} 22$ & $\mathrm{X} 23$ & $\mathrm{X} 24$ & $\mathrm{X} 25$ & $\mathrm{X} 26$ & $\mathrm{X} 27$ & $\mathrm{X} 28$ & $\mathrm{X} 29$ & \\
\hline Weight & 0.7196 & 0.6230 & 0.7457 & 0.7941 & 0.6909 & 0.9615 & 0.8521 & 0.7096 & 0.8569 & \\
\hline
\end{tabular}

If the number of indicators you choose is $\mathrm{M}$, when all the indicators for the red light, the overall score for the highest is $5 \mathrm{M}$, the lowest score is $\mathrm{M}$ when all the indicators for the blue light. Determine the limits of comprehensive early-warning index: take out $85 \%$ of $5 \mathrm{M}$ for the boundary of "red light" and "yellow light", take out 74\% and 50\% of 5M for the upper and lower boundaries of "green light", and take out $36 \%$ of $5 \mathrm{M}$ for the boundary of "light blue light" and "blue light". In our case, $\mathrm{M=29}$, the limits of comprehensive early-warning index are shown in Table 4.

Table 4 The limits of comprehensive early-warning index

\begin{tabular}{|c|c|c|c|c|c|}
\hline Early- warning state & overheating & steady & stable & decrease & shrinking \\
\hline Signal light & $\bullet$ & & $\bullet$ & $\bullet$ & $\bullet$ \\
\hline Interval limits & $>123.85$ & {$[107.3,123.85]$} & {$[72.5,107.3]$} & {$[52.2,72.5]$} & $<52.2$ \\
\hline
\end{tabular}

\section{Results and Discussion}

\subsection{The result and analysis of early-warning signal}

Through calculating according to the above value and limits of early-warning indicators, obtain the warning degree which showed by boom warning signal lights (Fig. 1) of environment-economy development of Dalian City. As shown in Figure 1, the whole status of environment-economy development transformed from the "light blue light" interval to the "green light" interval between 2005 and 2014, with the comprehensive early-warning index increasing gradually. From 2005 to 
2011, the overall stability is in the "light blue light" interval. It indicates that the environmental economic situation is getting cold during this period, the speed of development slow down, but the overall development is showing a rising state. Since 2012, the state of environment-economy development began to enter the "green light" interval, and has been on the rise until 2014. It indicates that the environmental economic system is stable, but there is still a trend of getting heater. Therefore, closely attention should be paid to the boom change of environmental economic development system, so as to take measures to prevent overheating of the system in time.

\begin{tabular}{|c|c|c|c|c|c|c|c|c|c|c|}
\hline & 2005 & 2006 & 2007 & 2008 & 2009 & 2010 & 2011 & 2012 & 2013 & \\
\hline $\mathbf{x} 1$ & $:$ & : & : & : & & & $\bullet$ & - & - & $\circ$ \\
\hline $\begin{array}{l}x_{2} \\
x_{3}\end{array}$ & 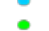 & . & 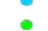 & $\bullet$ & 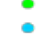 & 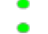 & 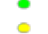 & 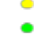 & 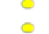 & • \\
\hline$x_{4}$ & • & • & $\bullet$ & $\bullet$ & • & • & • & $\bullet$ & 0 & $c$ \\
\hline xs & $\bullet$ & $\bullet$ & $\bullet$ & 0 & $\bullet$ & $\bullet$ & $\bullet$ & 0 & $\bullet$ & • \\
\hline$x_{6}$ & $\circ$ & • & • & • & $\bullet$ & • & $\bullet$ & $\bullet$ & 0 & c \\
\hline$x 7$ & • & • & • & $\bullet$ & • & • & • & 0 & 0 & $\circ$ \\
\hline $\mathrm{xs}$ & • & • & • & • & • & • & • & • & 0 & 。 \\
\hline xפ & • & • & • & • & • & $\bullet$ & $\bullet$ & $\bullet$ & $\circ$ & c \\
\hline $\mathbf{x} 10$ & • & $\bullet$ & • & $\bullet$ & $\bullet$ & $\circ$ & $\circ$ & $\bullet$ & $\bullet$ & $\bullet$ \\
\hline xi1 & $\circ$ & • & • & $\bullet$ & • & • & $\bullet$ & $\bullet$ & $\bullet$ & 0 \\
\hline $\mathrm{x} 12$ & • & • & • & • & • & • & $\circ$ & $\bullet$ & $\circ$ & 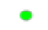 \\
\hline $\mathrm{x} 13$ & • & • & • & • & • & • & • & $\bullet$ & • & • \\
\hline $\mathrm{x} 14$ & • & • & • & • & • & • & $\bullet$ & $\bullet$ & $\bullet$ & $\bullet$ \\
\hline x1s & • & • & • & $\bullet$ & • & • & • & $\bullet$ & $\bullet$ & \\
\hline x16 & $\circ$ & $\circ$ & • & • & • & • & • & • & • & • \\
\hline $\mathbf{x 1 7}$ & • & • & • & • & 0 & $\circ$ & $\bullet$ & $\bullet$ & • & \\
\hline $\mathrm{X} 1 \mathrm{~s}$ & • & $\bullet$ & • & 0 & 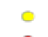 & ० & • & • & $\bullet$ & \\
\hline x19 & • & • & • & • & $\bullet$ & $\circ$ & • & $\circ$ & c & \\
\hline$x \geq 0$ & • & • & $\bullet$ & $\bullet$ & $\bullet$ & • & $\bullet$ & $\bullet$ & $\bullet$ & \\
\hline x21 & • & $\circ$ & • & • & $\bullet$ & • & $\bullet$ & $\bullet$ & $\bullet$ & \\
\hline$x 22$ & $\circ$ & • & ○ & • & $\bullet$ & $\bullet$ & $\bullet$ & $\bullet$ & $\bullet$ & \\
\hline$x 23$ & • & $\circ$ & • & • & • & $\bullet$ & $\bullet$ & $\bullet$ & • & \\
\hline $\mathrm{x}_{24}$ & • & • & • & • & • & $\bullet$ & $\bullet$ & $\bullet$ & $\circ$ & \\
\hline X2s & $\bullet$ & $\bullet$ & $\bullet$ & $\bullet$ & $\bullet$ & $\bullet$ & $\bullet$ & $\bullet$ & $\bullet$ & \\
\hline X26 & $\bullet$ & $\bullet$ & • & • & • & $\bullet$ & • & • & - & \\
\hline $\mathbf{x} 27$ & $\bullet$ & $\bullet$ & $\bullet$ & • & $\bullet$ & $\bullet$ & $\bullet$ & $\circ$ & - & \\
\hline$x_{2}$ & • & • & $\bullet$ & • & $\bullet$ & $\bullet$ & $\bullet$ & $\bullet$ & $\bullet$ & \\
\hline$x_{2}$ & $\bullet$ & $\bullet$ & $\bullet$ & $\bullet$ & $\bullet$ & $\bullet$ & $\bullet$ & $\bullet$ & 0 & 0 \\
\hline & 62.8 & 61.2 & 61.1 & $\alpha 3.52$ & 6.32 & ot.4 & 60.2 & 76.7 & 70.5 & \\
\hline $\begin{array}{l}\text { Nartang } \\
\text { dectree }\end{array}$ & $\bullet$ & $\bullet$ & 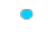 & 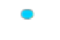 & $\bullet$ & $\bullet$ & $\bullet$ & $\bullet$ & $\bullet$ & \\
\hline
\end{tabular}

Fig. 1 The boom warning signal lights prediction

\begin{tabular}{|c|c|c|c|c|c|c|c|c|c|c|}
\hline & 2015 & 2016 & 2017 & 2018 & 2019 & 2020 & 2021 & 2022 & 2023 & 2024 \\
\hline$x_{1}$ & 0 & 0 & $\circ$ & 0 & 0 & 0 & $\bullet$ & 0 & 0 & 0 \\
\hline$x_{2}$ & $\bullet$ & $\circ$ & • & $\circ$ & $\circ$ & $\circ$ & $\circ$ & $\bullet$ & $\circ$ & • \\
\hline$x_{3}$ & 0 & • & • & • & • & $\circ$ & • & 0 & $\bullet$ & • \\
\hline$x 4$ & • & - & $\circ$ & $\circ$ & • & $\circ$ & 0 & 0 & 0 & - \\
\hline $\mathrm{xs}$ & • & • & • & • & • & $\circ$ & $\bullet$ & $\bullet$ & $\bullet$ & 이요 \\
\hline $\mathrm{x} 6$ & - & • & • & 0 & • & • & 0 & • & • & • \\
\hline$x 7$ & 。 & 。 & $\circ$ & • & $\bullet$ & • & $\circ$ & 0 & 0 & $\circ$ \\
\hline $\mathrm{xs}$ & ○ & • & $\circ$ & 。 & $\bullet$ & • & $\bullet$ & 0 & 0 & • \\
\hline $\mathrm{x} 9$ & - & ○ & $\circ$ & 0 & c & 0 & 0 & 0 & $\bullet$ & $\circ$ \\
\hline xio & $\bullet$ & • & $\circ$ & • & • & • & $\bullet$ & • & • & • \\
\hline xn & • & • & • & • & • & • & $\bullet$ & 0 & $\bullet$ & $\bullet$ \\
\hline $\mathrm{x} 12$ & • & $\bullet$ & $\circ$ & : & $:$ & - & $\bullet$ & : & $\bullet$ & : \\
\hline x13 & $\bullet$ & : & $:$ & : & 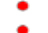 & ? & : & $\bullet$ & $\therefore$ & : \\
\hline $\begin{array}{l}x_{14} \\
x_{15}\end{array}$ & $:$ & : & $:$ & : & 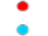 & : & $\dot{0}$ & $:$ & ? & : \\
\hline $\begin{array}{l}\text { xis } \\
\text { x16 }\end{array}$ & $\bullet$ & • & 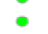 & • & 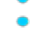 & $\bullet$ & $\bullet$ & 0 & $\bullet$ & 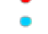 \\
\hline $\mathbf{x} 17$ & • & • & • & • & • & • & $\bullet$ & $\bullet$ & • & • \\
\hline X18 & $\bullet$ & • & $\circ$ & • & • & • & $\bullet$ & $\bullet$ & $\bullet$ & \\
\hline x19 & $\circ$ & • & $\circ$ & 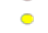 & • & 0 & 0 & 0 & 0 & 。 \\
\hline$x_{20}$ & • & • & • & • & • & • & $\bullet$ & $\bullet$ & $\bullet$ & • \\
\hline $\mathbf{x} 21$ & • & • & - & • & • & • & - & $\bullet$ & • & • \\
\hline$x_{2} z$ & $\bullet$ & • & - & • & • & • & $\bullet$ & $\bullet$ & $\bullet$ & • \\
\hline$x_{2}:$ & $\bullet$ & • & $\bullet$ & 。 & • & $\circ$ & $\bullet$ & • & • & - \\
\hline$x_{2}$ & 。 & • & $\bullet$ & - & $\circ$ & $\circ$ & • & 0 & • & • \\
\hline$x z$ & $\bullet$ & • & $\bullet$ & • & • & • & 0 & • & $\circ$ & • \\
\hline$x_{2}$ & 。 & $\circ$ & $\circ$ & • & $\circ$ & • & • & 0 & $\circ$ & 。 \\
\hline$x 27$ & 。 & $\circ$ & $\circ$ & • & • & • & 0 & 0 & • & $\circ$ \\
\hline $\mathrm{X} 2 \mathrm{~B}$ & $\bullet$ & • & - & • & • & • & • & • & 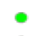 & • \\
\hline$x_{2}$ & $\bullet$ & • & $\bullet$ & 0 & 。 & $\bullet$ & 0 & 0 & 。 & $\circ$ \\
\hline & & & & & 77.4 & $8 \mathrm{ss}, 2$ & 80.7 & 80.4 & 78.2 & 96.7 \\
\hline $\begin{array}{l}\text { Wantatg } \\
\text { watar }\end{array}$ & 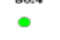 & 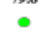 & 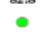 & 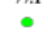 & 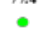 & $\cdot$ & • & • & $\bullet$ & 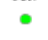 \\
\hline
\end{tabular}

Fig. 2 The boom warning signal lights of

\subsection{The result of early-warning system prediction}

Predicting the value of indicators in ten years based on the value's development trend between 2005 and 2014. It was divided into 8 groups from 2005 to 2014, and the data of six groups was selected as training data, with the data of 2014 as a test. In this study, R (10) is 4, it can be calculated that the hidden layer is 6 . The structure of the 3-6-1 BP neural network has been constructed, which satisfy the formula (4) and (5). Taking the index X1 (per capita GDP) as an example, the test results in 2014 were 111070 , the difference with true value of 109939 is $1.03 \%$, which was satisfactory. The comprehensive scores and warning degree has been calculated in the next ten years according to the predicted value of the indicators (limited to length, not a list), which showed by boom warning single lights (Fig. 2). In Fig. 2, the whole status of environment-economy development of Dalian City is stable and running in good condition in next ten years. But some indicators such as total social labor productivity, industrial smoke (dust) emissions, and industrial solid waste disposal rate and so on still need to be vigilant against to avoid overheating.

\section{Conclusion}

An early-warning system from the perspective of boom analysis was applied to evaluate the status of environment-economy development. We showed that the early-warning system has several good properties within this context: transparency, the use of qualitative evaluations, easy to understand, and traceability. In this paper, the indicator system was adjusted refer to the actual situation of Dalian city in the process of establishment which is enriched and optimized on the basis of predecessors. The early-warning system was introduced into the system of environment-economy development, pluralities of indicators were combined into a comprehensive index, and the results were showed by signal lights which are simple and clear. It is easy to analyze the development trend through analyzing the changes of signal lights. The BP neural network was used to predict indicators with the constant domestication of data according to the error curve, making the prediction results becoming 
more accurate. The early-warning system for evaluation has been developed respecting the theory of environment-economy development, so the final results can have a clear meaning in terms of environment-economy system. The above analysis shows that the environment-economy development of Dalian is in the stable state. The empirical results can better fits the present development of Dalian city, and provide decision makers with an effective basis for the formulation of development policies.

\section{Acknowledgments}

The authors acknowledge the financial support of the National Science and Technology Major Project (Water Pollution Control and Treatment Major Project (NO. 2012ZX07505-001-04)).

\section{References}

[1] Gao, F, Cao, MM, Ran, LI. A study on the yellow alert system of sustainable development of shaanxi province. J. Journal of Northwest University. 2007, 37(5), 947-851.

[2] Yu WJ, Zou XQ. Research on environmental early-warning mode of coastal newly development area in Jiangsu province. J. Resources \& Environment in the Yangtze Basin. 2007, 16(6), 775-780.

[3] Boggia, A., Cortina, C. Measuring sustainable development using a multi-criteria model: A case study. J. Journal of Environmental Management. 2010, 91(11), 2301-2306.

[4] Hao Q, Luo YM, Li LP. The sustainable development warning model of MEECS. J. Metal Mine. 2010, 2, 149-151.

[5] Wang Y, Ceng WH, Wu SZ, Jia JL. Elastic Coefficient-Based Warning System for the Environment-economic System of Daxing District. J. China Population, Resources and Environment. S1, 2011, 562-565.

[6] Dong J, Chi Y, Zou DA, Fu C, Huang Q, Ni MJ. Energy-environment-economy assessment of waste management systems from a life cycle perspective: Model development and case study. J. Applied Energy. 2013, 114(2), 400-408.

[7] Akinbami, J. F. K., Salami A. T., Siyanbola, W. O. An integrated strategy for sustainable forest-energy-environment interactions in Nigeria. J. Journal of Environmental Management. 2003, 69(2), 115-128.

[8] Wang Y, Ceng WH, Wu SZ. Analysis of environmental and economic situation based on the method of prosperity analysis. J. China Environmental Science. 2011, 09, 1571-1577.

[9] Zhang Q, Nie G, Hang S, Nan HU, Nan Z. Reliability robust design for cutting head of roadheader base on grey theory. J. Journal of Liaoning Technical University (Natural Science). 2013, 32(5), 668-671.

[10] Suresh S., Omkar S. N., Mani V. Parallel implementation of back-propagation algorithm in networks of workstations. J. Parallel and Distributed Systems, IEEE Transactions on. 2005, 16(1), 24-34.

[11] Hu, FY, Wang L, Wang SS, Liu XL, He GG. A Human Body Posture Recognition Algorithm Based on BP Neural Network for Wireless Body Area Networks. J. CHINA COMMUNICATIONS. 2016, 13(8), 198-208.

[12] Hou J. Construction of Sustainable Development Early Warning System and Its Empirical Research on Regional Economy. J. Science and Technology Management Research. 2014, 16, 55-61. [13] Li JL, Hu H, Jia DS. An Empirical Study on boom warning of Shanghai marine economy. J. Shanghai Management Science. 2015, 04, 85-90. 